\title{
The development and use of a pharmacist- specific Mini-CEX for postgraduate year trainees in Taiwan
}

\author{
Chung-Jen Wei ${ }^{1}$, Tzu-Hsuan Lu², Shu-Chen Chien ${ }^{3,4}$, Wan-Tsui Huang ${ }^{5}$, Yueh-Ping Liu' ${ }^{6}$, Cho-Yu Chan $^{7}$ \\ and Chiung-Hsuan $\mathrm{Chiu}^{5^{*}}$ (D)
}

\begin{abstract}
Background: Clinical pharmacists must have a complex combination of academic knowledge and practical experience that integrates all aspects of practice. Taiwan's Ministry of Health and Welfare in 2007 launched the Postgraduate Year (PGY) training program to increase the standard of pharmaceutical care. This study aims to develop a pharmacist-specific Chinese-language Mini-Clinical Evaluation Exercise (Mini-CEX) to evaluate the professional development of postgraduate year trainees.
\end{abstract}

Method: The specialized Mini-CEX was developed based on the core competencies of pharmacists, published literature, and expert opinion. A pilot test and evaluator workshop were held prior to the administration of the main test. Fifty-three samples were recruited. The main study was conducted at two regional teaching hospitals and a medical center teaching hospital in Taiwan between February and June 2012. The results were analyzed with the kappa statistic (inter-rater reliability) and descriptive statistics, while the Kruskal-Wallis test was used to examine the PGY trainees' Mini-CEX scores based on their performances.

Results: Trainees who had recently completed PGY programs (C-PGY) and 2nd year PGY trainees (PGY2) earned excellent scores, while the 1st year PGY trainees (PGY1) earned satisfactory scores in overall performance. C-PGY and PGY2 trainees also performed significantly better than PGY1 trainees in the organization and efficiency domain, and the communication skills domain.

Conclusion: This study demonstrates the feasibility of using the newly developed pharmacist-specific Chineselanguage version of the Mini-CEX instrument to evaluate the core competencies of PGY trainees in clinical settings.

Keywords: On-the-job training, Work-based assessment, Postgraduate year training program, Mini-CEX, PGY trainees

\section{Background}

Over the past few decades, the roles and obligations of hospital pharmacists around the globe have changed drastically. The healthcare industry places an ever-increasing emphasis on clinical work as a method for improving the safe, effective, and synergistic use of medication [1]. Clinical pharmacists have many responsibilities, including healthcare promotion, treatment of disease, and ensuring

\footnotetext{
* Correspondence: meg_chiu@tmu.edu.tw

This paper is submitted to BMC Medical Education.

${ }^{5}$ Department of Pharmacy. Cathay General Hospital, Taipei, Taiwan. School of

Pharmacy. Taipei Medical University, Taipei, Taiwan

Full list of author information is available at the end of the article
}

rational usage of pharmaceuticals by both healthcare professionals and patients.

According to the American College of Clinical Pharmacy, the competencies of clinical pharmacists are: to integrate therapeutic knowledge, problem-solving skills, judgment, and attitudes into service to meet the rising expectations of their teams and patients; to educate and communicate effectively with patients and healthcare professionals to ensure optimal patient outcomes; to maintain a continuously expanding and up-to-date knowledge base; to contribute to the development and implementation of protocols and critical pathways to manage patient populations; and to possess a therapeutic knowledge base of sufficient breadth and depth to

(c) The Author(s). 2019 Open Access This article is distributed under the terms of the Creative Commons Attribution 4.0 International License (http://creativecommons.org/licenses/by/4.0/), which permits unrestricted use, distribution, and reproduction in any medium, provided you give appropriate credit to the original author(s) and the source, provide a link to the Creative Commons license, and indicate if changes were made. The Creative Commons Public Domain Dedication waiver (http://creativecommons.org/publicdomain/zero/1.0/) applies to the data made available in this article, unless otherwise stated. 
effectively promote rational medication use [2]. These are the core competencies of appropriately educated pharmacists working within healthcare facilities. However, recent graduates often struggle to successfully translate theory into practice [3]. It is a crucial goal of new employee orientation and training to ensure that all entry-to-practice pharmacists can provide high-quality service. Bridging the gap between the required and equipped competencies of today's pharmacy graduates upon entry into the workplace is a prominent concern.

To ensure pharmacists' competence in all aspects of practice in order to provide holistic and patient-centered care, the Taiwanese government in 2007 launched the postgraduate year (PGY) program [4]. The Ministry of Health and Welfare also provided partial reimbursement to teaching hospitals to conduct training for 16 different groups of healthcare professionals, including pharmacists, nurses, and physical therapists, through the "Instruction Fee Reimbursement Program for Teaching Hospitals" to regulate on-the-job training. These programs are managed by the Joint Commission of Taiwan (JCT) under the supervision and instruction of the relevant professional associations. The purpose of the JCT PGY pharmacy program is to enhance the quality of comprehensive medical care by ensuring the acquisition of therapeutic knowledge, skills, attitudes, and behaviors over the course of the supervised 24-month training program. The program aims to equip trainee pharmacists with (1) proficient medication knowledge and evidence-based skills; (2) a patient-centered and total care attitude; (3) professional ethical reasoning and communication skills; and (4) ability to work in a team [5]. The teaching hospitals involved in the program develop their own training systems based on these primary objectives.

The American Board of Internal Medicine developed a focused, brief, and observed clinical encounter called the Mini-Clinical Evaluation Exercise (Mini-CEX). This encounter allows evaluators to directly observe the performance of a clinical task, and immediately follow up with a consultation between the trainee and evaluator, during which the evaluator completes an assessment and provides the trainee with feedback on their performance [6]. With the benefits of immediate constructive feedback and ongoing evaluation, the Mini-CEX is recognized as a useful tool for professional development and evaluating training program results, leading to improved clinical performance [7]. The Mini-CEX has been recognized by the Joint Programmes Board as a feasible and reliable method to assess the effectiveness of postgraduate pharmacy education [8].

Due to the effectiveness, reliability, and convenience of the Mini-CEX, it has been very popular for the evaluation of medical interns, residents, and other medical professionals in many Western countries as well as in Taiwan $[9,10]$. The role of pharmacists in the inpatient setting has expanded to emphasize client-centered and multi-disciplinary care. Although physicians and pharmacists often collaborate and share some common ground on patient care, many of the required competencies, attitudes, and practices differ significantly. Pharmacists are expected to play an active role in interprofessional care, while drawing on the knowledge, skills, and practices specific to their discipline [11], therefore, the direct application of the physician Mini-CEX to pharmacists is inappropriate.

There are a few pharmacist-specific Mini-CEXes, such as those developed by the Royal Pharmaceutical Society of Great Britain [12], the Department of Pharmacy at National Singapore University [13], and the Competency Development and Evaluation Group [14]. These tools focus on patient care, problem solving, and personal practice, but new pharmacists at hospitals in Taiwan are expected to perform more basic tasks, such as dispensing medication and providing consultation/education, while pharmacy divisions in most major hospitals deal with an average of 7000 outpatient visits per day. Only senior trainees are allowed to provide clinical pharmacy services after at least six months of PGY training. A work-based assessment of new pharmacists' core competencies that suits Taiwan's context is needed. Therefore, this study aimed to develop a Chinese-language Mini-CEX instrument that evaluates the professional development of pharmacists in PGY training programs in Taiwan.

\section{Methods}

\section{Study design}

This study was conducted to assess the competencies of new pharmacists participating in PGY training programs. A Chinese-language Mini-CEX was devised, tested, and utilized in six phases: (1) After a literature review and expert interviews, the instrument was developed; (2) An initial workshop for evaluators was held prior to the pilot test to insure inter-rater reliability; (3) The content of the instrument was re-evaluated and modified after a pilot test was conducted using a group of PGY trainees; (4) Workshops were held for evaluators before the main PGY trainee assessment; (5) A cross-sectional survey was conducted to measure the competencies of PGY trainees from the perspective of evaluators; (6) The core competencies of trainee groups were assessed and compared.

\section{Instrument development}

The specialized Mini-CEX for pharmacists was developed based on the core competencies of pharmacists outlined by the Canadian National Association of 
Pharmacy Regulatory Authorities [15], the accreditation standards for pharmacy residencies set by the American Society of Health-System Pharmacists (ASHP) [16], the clinical pharmacist competencies released by the American College of Clinical Pharmacy [2], and the pharmacist training guide produced by the Japan Pharmacists Education Center [17]. The ladder system established by the Taiwan Society of Health-System Pharmacists (TSHSP) [18] was also incorporated into the evaluation criteria. Nine core competencies were initially included in the instrument, which was validated by five experts: two MD/ $\mathrm{PhDs}$ specializing in instrument development and three PharmD-qualified directors of hospital pharmacy departments. Two rounds of the Delphi method were applied to check the face validity and the allocation of sample behavior items to the appropriate domains [19]. A three-point rating system was utilized to decide whether to include domains: domain is important and should be retained (3 points), domain is important but needs revision ( 2 points), and domain is not important and must be removed ( 1 point). The domains that scored an average of at least 2 points were retained.

The final version of the Mini-CEX included nine domains: pharmacology knowledge, patient care knowledge, consultation skills, professional health education skills, management of drug distribution, organization and efficiency, professionalism, communication skills, and overall performance (shown in Additional file 1). All descriptive items for each domain were adapted from guidelines provided by the ASHP, translated into Chinese, and validated using the Delphi method.

The complete evaluation form consisted of the evaluators' and trainee's identities, number of years of PGY training, the date and place of the evaluation, detailed descriptions of each domain, observations and feedback time, and the overall score. Descriptions of each domain are included in Additional file 2.

Trainees were scored based on the qualities of a "competent" pharmacist (equivalent to stage 3 of the TSHSP ladder system) $[18,20]$. Each core competency was rated on a 1-9 point scale as unsatisfactory (1-3), satisfactory (4-6), or excellent (7-9). Unsatisfactory scores (1-3) were defined as "extremely poor" (1 point), "poor" (2 points), and "nearly passing" (3 points). Satisfactory scores (4-6) were defined as "meets minimum expectations" (4 points), "average" ( 5 points), and "slightly above average" (6 points). Excellent scores (7-9) were defined as "meets most expectations and exceeds all others" (7 points), "exceeds most expectations and meets all others" (8 points), and "exceeds all expectations" ( 9 points). All behaviors were rated based on the observed practice for the selected case. Evaluators were advised to choose "not applicable" when certain behaviors were not performed.

\section{Participants and setting}

Since this study focused on the assessment of trainees based on the input of evaluators, two independent sets of evaluators and trainees were recruited for the pilot test and the main study. The qualifications required of evaluators and trainees were based on the requirements of the PGY program in Taiwan. Evaluators were required to have senior pharmacist status, at least four years of working experience in a teaching hospital, and instructor certification. The inclusion criteria for PGY trainees were: current participation in a PGY program or completion of PGY training within a year of the start of the study, and current employment in the pharmacy department of a teaching hospital.

\section{Pilot study and evaluator workshop}

The pilot test was held at two regional teaching hospitals and a medical center teaching hospital in Taiwan in December 2011 to confirm the appropriateness of the instrument. Thirteen PGY trainees and eight evaluators were recruited. Ten trainees were female $(76.9 \%)$ and three were male $(23.1 \%)$. The trainees reported a mean age of 24.38 (SD 2.21) and mean tenure of 6.33 months (SD 4.98) in PGY programs.

A two-session workshop was held for the evaluators prior to the pilot study to finalize the instrument and maintain the consistency of ratings for the same items between multiple respondents [21]. In the first session, the background, concept, purpose, and procedure of the Mini-CEX were reiterated. In the second session, a sample video clip of a pharmacist-patient Mini-CEX encounter was shown to the evaluators, who were asked to give a score for the encounter and provide a brief explanation to the other evaluators for the score given. An evaluator-hosted discussion was then held to establish performance standards and reach a consensus among the evaluators. Once a consensus was reached, the video clip was shown to the evaluators again for scoring. After analysis of the second-round evaluation results, the inter-rater reliability was determined.

\section{Main study}

The main study was conducted at two regional teaching hospitals and a medical center teaching hospital in Taiwan between February and June 2012. Fifty-three PGY trainees and 22 senior pharmacist evaluators were recruited using the same inclusion criteria as the pilot study, with an evaluator to trainee ratio of 1:2.4.

All evaluators followed the protocol outlined in the workshop. A trainee's performance was observed by two evaluators who rated them as compared with a TSHSP stage 3 pharmacist. One evaluator was a direct supervisor, while the other was a mentor of the trainee. These relationships were chosen to minimize subjectivity and 
enhance the quality of the assessment [22]. All behaviors were rated based on the observed practice in the selected settings, including dispensing medication, providing clinical pharmacy services, and giving a medication consultation. Technical skills, knowledge, and interaction with patients and peers were assessed. After asking the trainee for therapeutic decisions and reminders for therapeutic management, the evaluators completed the Mini-CEX form and provided "sandwich" feedback followed by direct instructions for improvement [23].

\section{Data analysis}

The results were analyzed in two parts using SPSS for Windows 22.0. Firstly, the kappa statistic (inter-rater reliability) was calculated based on the scores given by the evaluators before and after the discussion during the workshop. Secondly, descriptive statistics and the Kruskal-Wallis test were used to examine the PGY trainees' Mini-CEX scores.

\section{Ethics approval}

The Joint Institutional Review Boards of participating hospitals approved the study. The faculty members and PGY trainees were informed of the purpose of the study and assured of confidentiality. Written informed consent was obtained from the enrolled participants for purposes of publication of this report. The Joint Institutional Review Board of Taipei Medical University approved the study. The approval number is TMU 201012008.

\section{Results}

\section{Validity and reliability}

In this study, face validity was assessed by five experts. A two-round Delphi process was conducted to devise the instrument. After the evaluators' workshop, the inter-rater reliability of the instrument was obtained on the basis of the second-round evaluation. The inter-rater reliability of the instrument was 0.7 .

\section{Descriptive statistics}

The main study proceeded with 53 PGY trainees, including 1st year (PGY1) and 2nd year (PGY2) trainees, and pharmacists who had completed their PGY training within the past year (C-PGY). A total of 104 evaluations were collected with a $100 \%$ response rate (Table 1). The trainees had completed an average of 14.7 months of training, and PGY1 students comprised the largest proportion of the sample (47.2\%). They were mostly female (79.2\%), and the most common age group was 25 to 29 years (64.2\%). Trainees were observed dispensing medication for outpatients (55.4\%), dispensing medication for hospitalized patients (18.5\%), and providing clinical pharmacy services $(4.6 \%)$.
Table 1 Descriptive Statistics of PGY Trainees

\begin{tabular}{|c|c|c|}
\hline & Variable & n (\%) \\
\hline \multirow[t]{3}{*}{ Duration of Training 14.7 (11.3) } & 1st year (PGY1) & $25(47.2)$ \\
\hline & 2nd year (PGY2) & $20(37.7)$ \\
\hline & Recent graduate (C-PGY) & $8(15.1)$ \\
\hline \multirow[t]{2}{*}{ Gender } & Male & $11(20.8)$ \\
\hline & Female & $42(79.2)$ \\
\hline \multirow[t]{3}{*}{ Hospital $^{b}$} & A & $9(17.0)$ \\
\hline & B & $8(15.1)$ \\
\hline & C & $36(67.9)$ \\
\hline \multirow[t]{4}{*}{ Age 25.5 (1.9) } & $21-24$ & $15(28.3)$ \\
\hline & $25-29$ & $34(64.2)$ \\
\hline & Above 30 & $2(3.8)$ \\
\hline & Not reported & $2(3.8)$ \\
\hline
\end{tabular}

Note: Mean (SD); ${ }^{\text {a }}$ completed PGY training within the past year

${ }^{\mathrm{b}} \mathrm{A}$ (medical center); B (regional hospital); C (medical center)

The mean ratings for all 53 trainees were highest in the communication skills domain $(7.26 \pm 1.20)$ and lowest in the organization and efficiency domain $(6.79 \pm$ 1.16). The mean score in the overall performance domain was $7.07( \pm 1.17)$, indicating that the competencies of trainees enrolled in the study met the criteria for "excellent" performance (Table 2). In the subgroup analysis, PGY1 trainees performed best in pharmacology knowledge $(6.94 \pm 1.03)$, PGY2 trainees performed best in communication skills $(7.82 \pm 0.86)$, and C-PGY trainees performed best in medication consulting skills $(7.75 \pm 1.04)$.

A one-way ANOVA shows that C-PGY trainees performed significantly better $(P<0.05)$ than PGY1 trainees in the organization and efficiency domain, and the overall performance domain. Similarly, PGY2 trainees performed significantly better $(P<0.05)$ than PGY1 trainees in organization and efficiency, communication skills, and overall performance.

\section{Discussion}

The PGY training program is beneficial to the development of optimal pharmaceutical care for clinical pharmacists in Taiwan, as it encourages the integration of academic knowledge and clinical practice. This is especially important for new pharmacists who are entering the workforce, facing real patients, and taking responsibility for their clinical skills and decisions for the first time [24]. As healthcare development advances, the importance of continuous education and updating the undergraduate and graduate knowledge bases increases.

The study instrument included nine major competencies described by major professional pharmacy associations, and a two-round Delphi process was utilized to verify the face validity. The Delphi method is a feasible 
Table 2 Cross-Comparison of PGY Groups on Evaluation Dimensions

\begin{tabular}{|c|c|c|c|c|c|c|}
\hline Evaluation Dimensions & $\begin{array}{l}\text { Overall } \\
(N=53)\end{array}$ & $\begin{array}{l}\text { PGY1 } \\
(n=25)\end{array}$ & $\begin{array}{l}\text { PGY2 } \\
(n=20)\end{array}$ & $\begin{array}{l}\text { C-PGY } \\
(n=8)\end{array}$ & $p$ value & Post-hoc \\
\hline Pharmacology Knowledge & $7.1(0.9)$ & $6.9(1.0)$ & $7.5(0.4)$ & $7.1(0.3)$ & .12 & \\
\hline Patient Care Knowledge & $7.1(1.0)$ & $6.6(1.0)$ & $7.4(0.9)$ & $7.3(0.9)$ & .08 & \\
\hline Medication Consulting Skills & $7.0(1.0)$ & $6.6(1.1)$ & $7.0(1.4)$ & $7.7(1.0)$ & .17 & \\
\hline Health Professional Education Skills & $6.8(1.6)$ & $6.5(1.1)$ & $7.0(1.5)$ & $7.2(1.0)$ & .42 & \\
\hline Management of Drug Distribution & $6.9(1.1)$ & $6.5(1.2)$ & $7.1(1.4)$ & $7.3(0.6)$ & .13 & \\
\hline Organization and Efficiency & $6.7(1.1)$ & $6.2(1.1)$ & $7.1(1.2)$ & $7.2(0.7)$ & .03 & $\mathrm{PGY} 2>\mathrm{PGY} 1^{*} ; \mathrm{C}-\mathrm{PGY}>\mathrm{PGY} 1^{*}$ \\
\hline Professionalism & $7.0(1.1)$ & $6.5(1.2)$ & $7.5(0.8)$ & $7.5(0.8)$ & .07 & \\
\hline Communication Skills & $7.2(1.0)$ & $6.6(1.2)$ & $7.8(0.8)$ & $7.5(1.1)$ & .00 & $\mathrm{PGY} 2>\mathrm{PGY} 1^{*}$ \\
\hline Overall Performance & $7.0(1.1)$ & $6.5(1.3)$ & $7.4(0.8)$ & $7.6(0.6)$ & .01 & $P G Y 2>P G Y 1 *$; $-P G Y>P G Y 1 *$ \\
\hline Observation Time & $14.3(10.1)$ & $12.9(7.6)$ & $17.0(13.7)$ & $12.5(5.1)$ & .40 & \\
\hline Feedback Time & $6.7(4.1)$ & $7.5(4.6)$ & $6.3(4.4)$ & $5.6(0.8)$ & .58 & \\
\hline
\end{tabular}

Note: Mean (SD), C-PGY = Completed PGY training within the past year

way to reach consensus, especially for pharmacist competency studies [25]. The nine competency domains listed in the Mini-CEX represent the skills pharmacists must have. They also align with the ladder systems for clinical pharmacists and the Good Pharmacy Practice guidelines $[18,26]$. These guidelines, released by the TSHSP, describe the standards used to assess the quality of pharmacy practice and provide the basis of current and future practice-support initiatives.

The Mini-CEX is a recognized evaluation method with strong validity [27] that has frequently been utilized for both interns and residents across medical specialties. As opposed to the written evaluations that typically bookend the PGY program, this assessment of actual patient encounters through direct observation can provide ongoing data on trainee performance. The Mini-CEX is a workplace-based assessment that fosters the type of self-directed learning environment essential for continuing professional development [28]. A Mini-CEX typically includes $15 \mathrm{~min}$ of observation and $5 \mathrm{~min}$ of feedback [6]. In our study, the mean observation and feedback times were 14.30 and $6.79 \mathrm{~min}$, respectively. The feedback time was relatively long due to its structured nature, as evaluators were instructed to provide detailed recommendations, and trainees were encouraged to respond. There was no significant difference in feedback times between the three groups. The provision of feedback after direct observation is a critical component of deliberate practice, a learning strategy believed to maximize the improvement of clinical competency through repetition, guidance, and coaching [29].

There were eight evaluators in the evaluator workshop, and the instrument showed acceptable reliability (inter-rater reliability of 0.7). Although the physician Mini-CEX has been proven to successfully differentiate between competency levels with appropriate reliability and construct validity [30], some studies have demonstrated limitations of the Mini-CEX due to significant inter-evaluator variability [31]. Medical educators typically evaluate trainees based on personal experience and performance. Formal training is needed to minimize the variability between evaluators and to reach consensus. A study by Liao et al. demonstrated that faculty participation in Mini-CEX workshops strengthened the consistency of evaluators and resulted in the implementation of a successful Mini-CEX assessment program [32]. Meanwhile, a study by Arora et al. found that using standardized video-based scenarios depicting differing levels of performance, communication, and professionalism in a variety of settings ensured valid and reliable physician handoff Mini-CEX [33]. Moreover, a meta-analysis of non-medical education studies suggested that training workshops for raters were associated with score accuracy improvement. This is also important for evaluator professional development, as the literature has highlighted positive impacts on personal learning and performance from serving as a Mini-CEX evaluator [34].

The goal of assessing the core competencies of PGY trainees, as demonstrated by how they interacted with patients during clinical encounters, was achieved using a customized pharmacist Mini-CEX. The results for each trainee can be considered with regard to the learning curve and desired teaching outcomes of their program. Based on the individual results, trainees and teachers can adjust their approaches to lessons, behavior, and attitudes to achieve better learning outcomes.

The results of this study revealed the successes of PGY training programs at all three participating institutions. The average scores in the "overall performance" domain for all trainee groups were high: average PGY1 scores were "satisfactory," while average PGY2 and C-PGY scores were "excellent." Notably, every C-PGY trainee 
scored a 7.0 (the threshold for an "excellent" score) or higher in overall performance. This was consistent with the results of other studies evaluating PGY trainee training outcomes in Taiwan [35].

Mean scores for all nine competency domains were higher for C-PGY trainees than PGY1 pharmacists. In addition to the overall performance domain, two other domains showed statistically significant higher scores for the senior groups: the organization and efficiency domain, and the communication skills domain. These results demonstrate the importance of postgraduate on-the-job training and indicate that the development and enhancement of these abilities requires actual practice in the workplace, such as interaction with other medical professionals and participation in multidisciplinary teams.

Our study reveals significant differences in organization and efficiency, as well as communication skills of trainees undertaking PGY programs. Based on the outcomes of the JCT's 24-month PGY training program, organization and efficiency, as well as communication skills could be improved with training in multidisciplinary teamwork, as well as social and administrative pharmacy [5]. In most instances, the trainees could enroll in these elective courses only if they were already performing well after basic training and the courses are provided by qualified faculty. We suggest that social and administrative pharmacy, as well as interprofessional practice be listed as required subjects in the Taiwanese PGY training program, as it is in many pharmacist residency programs in the United States [10]. In the meantime, pharmaceutical education policy should encourage teaching hospitals to improve the quality of instruction in required courses to facilitate better patient care and faster acquisition of clinical skills.

\section{Limitations}

This study has three limitations: First, the research was conducted at one regional hospital and two medical centers in northern Taiwan. Although these institutions were teaching hospitals accredited by the JCT, the quality of teaching and the format of the PGY program differs between hospitals and regions. The performance of pharmacists throughout the national PGY program requires further evaluation. Second, this cross-sectional study only represents the ability of trainees at the time of the study. To evaluate individual growth through participation in the PGY program, advanced longitudinal studies are required. Third, a major advantage of formative evaluation methods in training programs is their ability to help modify clinical practice following the evaluation and feedback process. Unfortunately, the changes in knowledge and behavior following the Mini-CEX were not able to be measured as part of this study.

\section{Conclusion}

This study developed a Chinese-language PGY trainee version of the Mini-CEX instrument. The instrument was based on the structure of the previously validated and widely used Mini-CEX for medical residents [6]. The specific elements of this Mini-CEX for pharmacists were developed based on clinical practice and educational guidelines, published literature, and expert opinion. This process resulted in nine domains for PGY trainee assessment: pharmacology knowledge, patient care knowledge, medication consulting skills, professional health education skills, management of drug distribution, organization and efficiency, professionalism, communication skills, and overall performance. We believe that these adjustments have created an assessment tool that is more compatible with the core competencies of pharmacists. It should serve as a practical workplace-based assessment for educators to evaluate a trainee's strengths and weaknesses, and to give timely formative feedback.

The results demonstrate that the Mini-CEX is a feasible tool to evaluate the professional development of pharmacists. Trainee scores indicated the merit of their institutions' PGY training programs. Additionally, the senior trainees performed significantly better in the core competencies of overall performance, organization and efficiency, and communication skills, which demonstrates the importance of post-graduate onsite training and interaction with other health professionals. Multidisciplinary team participation, and social and administrative pharmacy should be listed as required subjects in the Taiwanese PGY training program.

Our Mini-CEX is feasible for use in clinical settings for the evaluation of the core competencies of PGY trainees. Nevertheless, further longitudinal studies using this tool are recommended to assess personal growth and the development of expertise during the PGY training period and beyond.

\section{Additional files}

Additional file 1: Pharmacist Mini-CEX Evaluation Sheet. (DOCX 23 kb)

Additional file 2: Definitions of major domains. (DOCX $16 \mathrm{~kb}$ )

\section{Abbreviations \\ ASHP: The American Society of Health-System Pharmacists; C-PGY: Those who have completed their PGY in the past year; JCT: Joint Commission of Taiwan; Mini-CEX: Mini-Clinical Evaluation Exercise; PGY: Postgraduate Year; PGY1: First-year postgraduate trainees; PGY2: Second-year postgraduate trainees; TSHSP: The Taiwan Society of Health-System Pharmacists}

\section{Acknowledgements}

The authors appreciate the support of the faculty members and trainees who participated in this study. Most of all, we would like to thank Dana A. Jensen and Kayleigh Madjar for their editing services and assistance with this manuscript. 


\section{Funding}

This study was supported by the Ministry of Health and Welfare, which financially supported the research design, data collection, analysis, and interpretation of data. Grant numbers were DOH100-TD-M ${ }^{-113}-100007$ (1 of 2) and DOH101-TD-M ${ }^{-113}-100007$ (2 of 2). There was no additional external funding.

\section{Availability of data and materials}

No data has been submitted to any open-access databases. All data supporting the study are presented in the manuscript or are available upon request.

\section{Author's contributions}

CHC, CJW, and YPL designed, conceived of, and did research for the study. All authors carried out the study, prepared the instrument, and collected data. CHC performed statistical analysis. All authors prepared the manuscript. CJW and THL contributed to the manuscript equally, and deserved to be listed as first authors. CHC and THL responded to editorial and reviewers' comments. All authors read and approved the final manuscript.

\section{Ethics approval and consent to participate}

The Ethics Committee of Taipei Medical University approved the study protocol (approval number TMU201012008). Informed written and oral consent was obtained from all participants prior to participation in the study.

\section{Consent for publication}

Not applicable.

\section{Competing interests}

The authors declare no potential competing interests with respect to the research, authorship, and/or publication of this article.

\section{Publisher's Note}

Springer Nature remains neutral with regard to jurisdictional claims in published maps and institutional affiliations.

\section{Author details}

'Department of Public Health, Fu Jen Catholic University, New Taipei City, Taiwan. ${ }^{2}$ Medical Quality Department, Taipei Medical University-Shuang Ho Hospital, New Taipei City, Taiwan. ${ }^{3}$ School of Pharmacy, College of Pharmacy, Taipei Medical University, Taipei, Taiwan. ${ }^{4}$ Department of Pharmacy, Taipei Medical University Hospital, Taipei, Taiwan. ${ }^{5}$ Department of Pharmacy. Cathay General Hospital, Taipei, Taiwan. School of Pharmacy. Taipei Medical University, Taipei, Taiwan. ${ }^{6}$ Department of Emergency Medicine, National Taiwan University Hospital, Taipei, Taiwan. ${ }^{7}$ Changhua Christian Hospital, Chunghua, Taiwan.

Received: 11 February 2019 Accepted: 13 May 2019

Published online: 22 May 2019

\section{References}

1. Lee JK, Slack MK, Martin J, Ehrman C, Chisholm-Burns M. Geriatric patient care by U.S. pharmacists in healthcare teams: systematic review and metaanalyses. J Am Geriatr Soc. 2013;61:1119-27.

2. Burke JM, Miller WA, Spencer AP, Crank CW, Adkins L, Bertch KE, Ragucci DP, Smith WE, Valley AW. Clinical pharmacist competencies. Pharmacotherapy. 2008;28:806-15.

3. Woodard LJ, McKennon S, Danielson J, Knuth J, Odegard P. An elective course to train student pharmacists to deliver a community-based group diabetes prevention program. Am J Pharm Educ. 2016;80:106.

4. Chu TS, Weed HG, Yang PC. Recommendations for medical education in Taiwan. J Formos Med Assoc. 2009;108:830-3.

5. Joint Commission of Taiwan. Guidelines for the 2-year Medical Vocational Training Course: Joint Commission of Taiwan. 2012. http://www.tjcha.org. tw/FrontStage/page.aspx?ID=5DDE57A5-EE32-47DC-B4EEBD70E32522F7\&PID=F119DC8B-752D-4971-A868-C109A44118C5. Accessed 22 Mar 2015.

6. Norcini JJ, Blank LL, Duffy FD, Fortna GS. The mini-CEX: a method for assessing clinical skills. Ann Intern Med. 2003;138:476-81.

7. Gauthier S, Cavalcanti R, Goguen J, Sibbald M. Deliberate practice as a framework for evaluating feedback in residency training. Med Teach. 2015; 37:551-7.
8. Holmboe ES, Sherbino J, Long DM, Swing SR, Frank JR. The role of assessment in competency-based medical education. Med Teach. 2010; 32(8):676-82.

9. Kim S, Willett LR, Noveck H, Patel MS, Walker JA, Terregino CA. Implementation of a mini-CEX requirement across all third-year clerkships. Teach Learn Med. 2016:28:424-31.

10. Chen W, Lin CC, Huang CC, Tsai CH, Tsai CH. Implementation of the miniCEX (clinical evaluation exercise): experiences and preliminary results. J Med Education. 2006:10:232-9.

11. World Health Organization. The role of the pharmacist in the health care system. 1994. http://apps.who.int/medicinedocs/pdf/h2995e/h2995e.pdf. Accessed 22 Aug 2018.

12. Rayal Pharmaceutical Society of Great Britain. Pharmaceutical Care Assessment (PCA). 2019. https://www.rpharms.com/development/ education-development/development-programmes/foundationprogramme/pharmaceutical-care-assessment-pca. Accessed 5 Apr 2019

13. Department of Pharmacy at of National Singapore University. National Collaborative Prescribing Programme-Mini Clinical Evaluation Exercise (MiniCex). 2018. http://pharmacy.nus.edu.sg/wp-content/uploads/2018/01/CPPMini-CEX-Form-v1.0.pdf. Accessed 5 Apr 2019.

14. The Competency Development and Evaluation Group. Mini-CEX (clinical evaluation exercise) for general level pharmacists. http://www.codeg.org/ fileadmin/codeg/pdf/Assessment_tool_instructions.pdf Accessed 5 Apr 2019

15. National Association of Pharmacy Regulatory Authorities. Professional Competencies for Canadian Pharmacists at Entry to Practice: National Association of Pharmacy Regulatory Authorities. 2014. http://napra.ca/ content_files/files/comp_for_cdn_pharmacists_at_entrytopractice_ march2014_b.pdf. Accessed 6 Oct 2015.

16. American Society of Health-System Pharmacists. Residency Program Directors: American Society of Health-System Pharmacists. 2010. https:// www.ashp.org/Professional-Development/Residency-Information/ResidencyProgram-Directors. Accessed 22 Nov 2016.

17. The Japan Pharmacists Education Center. Pharmacist continuing education guidelines: the Japan pharmacists education center. 2009. http://www.jpec. or.jp/. Accessed 23 May 2011.

18. Taiwan Society of Health-System Pharmacists. The ladder systems for clinical pharmacists. Taiwan. 2011. http://www.tshp.org.tw/ehc-tshp/s/ viewDocument?documentld=252736cae9ca4d5daa3b4772a7cbf9c1. Accessed 12 Feb 2018.

19. Linstone HA, Turoff M. The Delphi method: techniques and applications: Addison-Wesley Reading; 1975.

20. Dreyfus SE, Dreyfus HL. A five-stage model of the mental activities involved in directed skill acquisition: California Univ Berkeley Operations Research Center; 1980.

21. Litwin MS, Fink A. How to measure survey reliability and validity: Sage; 1995

22. Lee V, Brain K, Martin J. From opening the 'black box' to looking behind the curtain: cognition and context in assessor-based judgements. Adv Health Sci Educ Theory Pract. 2019;24(1):85-102.

23. Cantillon P, Sargeant J. Teaching rounds: giving feedback in clinical settings. BMJ. 2008;337(7681):1292-4.

24. Marshall JM, Adams JP, Janich JA. Practical, ongoing competencyassessment program for hospital pharmacists and technicians. Am J Health Syst Pharm. 1997;54:1412-7.

25. Bzowyckyj AS, Janke KK. A consensus definition and core competencies for being an advocate for pharmacy. Am J Pharm Educ. 2013;77:24.

26. Joint FIP/WHO guidelines on good pharmacy practice: standards for quality of pharmacy services. https://www.fip.org/www/uploads/database_file. php?id=331\&table_id=. Accessed 15 May 2019.

27. Kogan JR, Holmboe ES, Hauer KE. Tools for direct observation and assessment of clinical skills of medical trainees: a systematic review. JAMA. 2009;302:1316-26.

28. Liu C. An introduction to workplace-based assessments. Gastroenterol Hepatol Bed Bench. 2012;5:24-8.

29. Issenberg SB, McGaghie WC. Clinical skills training-practice makes perfect. Med Educ. 2002;36:210-1.

30. Holmboe ES, Huot S, Chung J, Norcini J, Hawkins RE. Construct validity of the miniclinical evaluation exercise (miniCEX). Acad Med. 2003;78:826-30.

31. Govaerts MJ, Van de Wiel MW, Schuwirth LW, Van de Vleuten CP, Muijtjens AM. Workplace-based assessment: raters' performance theories and constructs. Adv Health Sci Educ Theory Pract. 2013;18:375-96. 
32. Liao KC, Pu SJ, Liu MS, Yang CW, Kuo HP. Development and implementation of a mini-clinical evaluation exercise (mini-CEX) program to assess the clinical competencies of internal medicine residents: from faculty development to curriculum evaluation. BMC Med Educ. 2013;13:31.

33. Arora VM, Berhie S, Horwitz LI, Saathoff M, Staisiunas P, Farnan JM. Using standardized videos to validate a measure of handoff quality: the handoff mini-clinical examination exercise. J Hosp Med. 2014;9:441-6.

34. Chen W, Lai MM, Li TC, Chen PJ, Chan CY, Lin CC. Professional development is enhanced by serving as a mini-CEX preceptor. J Contin Educ Heal Prof. 2011;31:225-30.

35. Yeh FY, Dai SH, Chang HC. Using mini-CEX to evaluate teaching results and promote pharmacy service. Formos J Clinic Pharma. 2015;23:288-95.

Ready to submit your research? Choose BMC and benefit from:

- fast, convenient online submission

- thorough peer review by experienced researchers in your field

- rapid publication on acceptance

- support for research data, including large and complex data types

- gold Open Access which fosters wider collaboration and increased citations

- maximum visibility for your research: over $100 \mathrm{M}$ website views per year

At $B M C$, research is always in progress.

Learn more biomedcentral.com/submissions 